\title{
The oral administration of silver nanoparticles activates the kynurenine pathway in rat brain independently of oxidative stress
}

\author{
Oluyomi Stephen Adeyemi ${ }^{\mathrm{a}, *}$, Rhoda Ananu Uloko ${ }^{\mathrm{a}}$, Oluwakemi Josephine Awakan ${ }^{\mathrm{a}}$, \\ Anne Adebukola Adeyanju ${ }^{\mathrm{b}}$, David Adeiza Otohinoyi ${ }^{\mathrm{c}}$ \\ ${ }^{a}$ Medicinal Biochemistry, Nanomedicine and Toxicology Laboratory, Department of Biochemistry, Landmark University, PMB 1001, Km 4, Ipetu Road, Omu-Aran, \\ 251101, Nigeria \\ ${ }^{\mathrm{b}}$ Department of Biological Sciences, McPherson University, Seriki-Sotayo, Ogun State, Nigeria \\ ${ }^{\mathrm{c}}$ School of Medicine, All Saints University, Hillsborough Street, Roseau, Dominica
}

\section{A R T I C L E I N F O}

\section{Keywords:}

Inorganic nanoparticles

Medicinal biochemistry

Nanomedicine

Nanotoxicity

Reactive oxygen species

Tryptophan metabolism

\begin{abstract}
A B S T R A C T
In this work, we determined whether oxidative stress contributed to the activation of the kynurenine pathway by AgNPs. Male Wistar rats weighing between 130 and $146 \mathrm{~g}$ were randomly assigned into six groups. Animals in the negative control group were orally administered distilled water while, the other treatment groups were respectively given AgNPs ( 25 and $50 \mathrm{mg} / \mathrm{kg}$ bw) alone or in combination with Trolox (100 mg/kg bw). Results showed that treatments with AgNPs significantly raised protein carbonyl level in rat liver, but the co-treatment with Trolox attenuated the elevation. Conversely, AgNPs raised the level of reduced glutathione (GSH) in rat plasma and tissues compared to the negative control. Further, oral exposure to AgNPs ( $50 \mathrm{mg} / \mathrm{kg}$ bw) significantly elevated rat plasma and brain kynurenine levels compared to the negative control. Meantime, the cotreatment with Trolox appreciably restored kynurenine level in rat plasma, but not in the rat brain. Taken together, findings indicate that the oral administration of AgNPs alone at the doses used in this study, might not have caused oxidative stress. However, the co-treatment with Trolox appears to potentiate oxidative stress in rats following exposure to AgNPs. Furthermore, data support that the activation of the kynurenine pathway in the rat brain by AgNPs might be independent of oxidative stress. The findings are new and contribute to deepen our understanding of the cellular interaction by nanoparticles.
\end{abstract}

\section{Introduction}

The use of nanoparticles and/or nanomaterials is rapidly expanding. Nanoparticles are being deployed for several biomedical applications; drug delivery systems, cellular targeting and imaging, fluorescence imaging and as antibacterial or antimicrobial agents [1-3]. For example, the silver nanoparticles are known to have antibacterial activities and can therefore be used as antibacterial agents or as broadspectrum antimicrobials against bacteria, viruses and other microorganisms $[4,5]$. Interestingly, the small size and the large ratio of surface area to the volume of these nanoparticles make them more reactive, and they can easily penetrate cells and interact with biomolecules in a number of ways [6,7]. In the event of cellular uptake, nanoparticles can interact with several cellular biomolecules leading to oxidative stress, inflammatory response and/or apoptosis [8,9]. That nanoparticles facilitate the generation of free radicals, particularly reactive oxygen species (ROS) with potential to cause oxidative damage of cellular materials is well reported [1,10]. Nevertheless, our knowledge and understanding of the cellular interactions and/or effect by nanoparticles are still infant. However, emerging evidence indicates that inorganic nanoparticles particularly silver and gold nanoparticles have multiple cellular targets among which is the activation of the kynurenine pathway (the degradation product of L-tryptophan metabolism) [11].

Briefly, oxidation of L-tryptophan to kynurenine is catalyzed by the indoleamine 2,3-dioxygenase (IDO), while the non-enzymatic cyclization of kynurenine forms the quinolinate, which in turn can be converted to niacin. On the other hand, L-tryptophan can be transformed to form serotonin (neurotransmitter), which can be metabolized further to produce melatonin (neuro-hormone) [12]. Investigations have revealed that oxidative stress resulting from ROS production can affect the immune activity and neurotransmitter levels by influencing neurotransmitter synthesis [13]. Free radicals can stimulate the adaptive immune response in manners that may significantly impact on the L-

\footnotetext{
* Corresponding author.

E-mail address: yomibowa@yahoo.com (O.S. Adeyemi).
} 
tryptophan metabolism. During the adaptive immune response, there is an increase in pro-inflammatory cytokines and one of the functions of these immune messengers is to increase activity of IDO. When IDO activity is increased, there is less L-tryptophan available for making serotonin. Low serotonin levels have been associated with clinical symptoms such as depression, insomnia, and anxiety. Together, these facts linked oxidative stress to the activation of the kynurenine pathway [13], however, we do not know yet how these cellular targets relate in the presence of nanoparticles. Our understanding would be enhanced if we know how nanoparticles activate the kynurenine pathway. Therefore, this work attempted to determine whether AgNPs activate the kynurenine pathway by causing oxidative stress in Wistar rats.

\section{Materials and methods}

\subsection{Chemical and reagents}

Silver nanoparticles (AgNPs) was gifted by the Nanomedicine Research Group at the National Research Center for Protozoan Diseases (NRCPD), Obihiro University of Agriculture \& Veterinary Medicine, Obihiro, Japan. Trolox, kynurenine standard and Ehrlich reagents were products of Sigma (St. Louis, MO, USA). All other reagents were of analytical grade and used as supplied.

\subsection{Experimental animals}

Twenty-four male Wistar rats with an average weight between 130 and $146 \mathrm{~g}$ were used for this study. Animals were sourced from the Department of Biochemistry, University of Ilorin, Ilorin, Nigeria. The rats were housed in plastic cages under a well-ventilated condition and allowed to acclimate for two weeks before the commencement of the study. Rats were fed with the commercial rat pellet and were given unlimited access to clean water.

\subsubsection{Ethics statement}

Handling and treatment of animals were humane and consistent with the International Guiding Principles for Biomedical Research Involving Animals, Geneva, Switzerland [14]. This was under the observation of the local Institutional Ethics Committee on Scientific Research with approval and protocol 05022018.

\subsubsection{Animal grouping and treatment protocol}

The animals were randomly distributed into six (6) groups of four (4) rats in each group. The rats were given oral administration of silver nanoparticles singly or in combination with Trolox. Further details of the treatment protocols are shown below;

Negative control: Administered $1 \mathrm{ml}$ of distilled water.

AgNPs $25 \mathrm{mg} / \mathrm{kg}$ : Administered AgNPs at $25 \mathrm{mg} / \mathrm{kg}$ bw only.

AgNPs $50 \mathrm{mg} / \mathrm{kg}$ : Administered AgNPs at $50 \mathrm{mg} / \mathrm{kg}$ bw only.

AgNPs 25 mg/kg + TRO: Administered AgNPs ( $25 \mathrm{mg} / \mathrm{kg}$ bw) plus Trolox (100 mg/kg bw).

AgNPs 50 mg/kg + TRO: Administered AgNPs (50 mg/kg bw) plus Trolox (100 mg/kg bw).

TRO $100 \mathrm{mg} / \mathrm{kg}$ : Administered Trolox (100 mg/kg bw) only.

Silver nanoparticles were prepared in distilled water and the treatment was daily for seven days. The selection of doses was premised on previous studies $[1,15]$.

\subsubsection{Preparation of plasma and tissue homogenates}

Animals were fasted overnight after the last treatment and were sacrificed under mild anesthesia using diethyl ether. Blood samples from the rats were collected in clean EDTA bottles and these were centrifuged using a table-top centrifuge (model C5, LW Scientific, GA, USA) at $5000 \mathrm{rpm}$ for $10 \mathrm{~min}$ to yield plasma. The plasma was collected in plain sample bottles and stored frozen until needed for biochemical analysis. The brain and liver samples were excised, cleaned and homogenized in ice-cold $0.25 \mathrm{M}$ sucrose solution. The tissue homogenates were used for the determination of biochemical indices.

\subsection{Biochemical assays}

Biochemical determinations in rat plasma and tissue homogenates were carried out on a UV/Vis spectrophotometer (Jenway, Staffordshire, United Kingdom) where applicable. The total protein was determined by using the method described by Gornall et al. [16], while protein carbonyl level was estimated by the method described by Castegna et al. [17]. Malondialdehyde as a byproduct of lipid peroxidation was assayed as previously described by Varshney and Kale [18]. The kynurenine level was determined as previously reported elsewhere [11]. The level of reduced glutathione (GSH) was determined by using the method described by Bentler et al. [19], while the diphenylalanine assay (DPA) was used to determine DNA fragmentation as previously reported elsewhere [20].

\subsection{Statistical analysis}

Data were analyzed using the one-way ANOVA (GraphPad Software Inc., San Diego, CA, USA) and presented as mean value of four replicates \pm standard error of mean (SEM). Differences among the group mean values were determined by the Tukey's post-hoc test. Mean values at $p<0.05$ were considered to be significant.

\section{Results}

\subsection{AgNPs showed no detectable effect on rat average weight}

The oral administration of AgNPs as well as the co-treatment with Trolox had no appreciable effect on average weight of rats when compared to the negative control. The rat average weight before and after the treatment courses was not significantly different (Fig. 1a and b).

\subsection{AgNPs mildly altered rat brain and liver total protein levels}

The oral treatment at both low and high doses of AgNPs as well as in the combined treatment with Trolox did not significantly affect rat plasma total protein (Fig. 2a). However, total protein levels in rat brain and liver were decreased $(p<0.05)$ following oral exposure to AgNPs $(50 \mathrm{mg} / \mathrm{kg} \mathrm{bw})$, but were restored by the co-treatment with Trolox (Fig. 2b and c).

\subsection{AgNPs activated the kynurenine pathway in rat brain independently of oxidative stress and/or ROS production}

The AgNPs appear to cause a dose-dependent increase in kynurenine levels in rat brain and plasma, but showed no detectable effect on the rat liver kynurenine level (Fig. 3a-c). In particular, oral exposure to AgNPs $(50 \mathrm{mg} / \mathrm{kg}$ bw) elevated $(p<0.05)$ rat brain and plasma kynurenine levels. Interestingly, oral exposure to AgNPs ( $50 \mathrm{mg} / \mathrm{kg} \mathrm{bw})$ in combination with Trolox $(100 \mathrm{mg} / \mathrm{kg}$ bw) successfully modulated the elevation of kynurenine in rat plasma and not in the brain. This may likely indicate exclusion of oxidative stress in the activation of the kynurenine pathway by AgNPs in the rat brain.

\subsection{AgNPs mildly elevated oxidative stress indices}

In order to evaluate whether AgNPs caused oxidative stress, protein carbonyl and malondialdehyde (the measure of lipid peroxidation) were determined in rat plasma and tissues. Oral exposure to AgNPs mildly caused a dose-dependent increase in rat plasma protein carbonyl levels compared to the negative control (Fig. 4a). Meanwhile, the cotreatment with Trolox appeared to potentiate the capacity of AgNPs to 

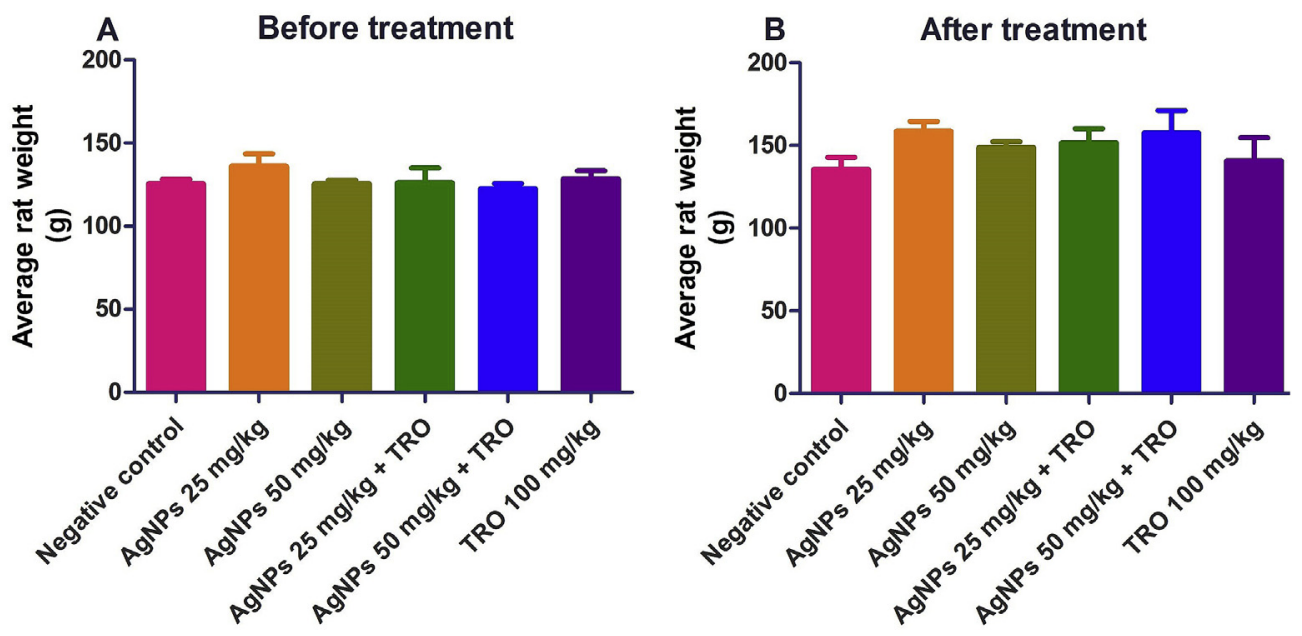

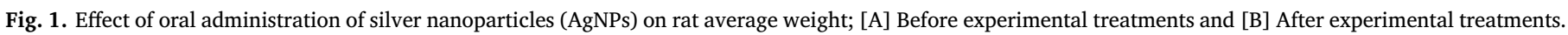
Data are presented as mean value of four replicates \pm standard error of mean (SEM).

elevate rat plasma protein carbonyl levels. In rat liver, protein carbonyl level was inconsistently altered; AgNPs $(25 \mathrm{mg} / \mathrm{kg}$ bw) caused a significant increase in protein carbonyl level, but this was restored by the co-treatment with Trolox (Fig. 4b). In rat brain, oral exposure to AgNPs alone decreased protein carbonyl level compared to the negative control (Fig. 4c). But again, the co-treatment with Trolox potentiated the capacity of AgNPs to elevate protein carbonyl level.

The oral exposure to only AgNPs at both low and high doses had no appreciable effect on malondialdehyde level in rat plasma compared with the negative control (Fig. 5a). However, the co-treatment with Trolox increased malondialdehyde level for AgNPs ( $50 \mathrm{mg} / \mathrm{kg}$ bw). The oral exposure to either AgNPs alone or the co-treatment with Trolox inconsistently altered rat liver and brain malondialdehyde levels, though these alterations were not statistically significant (Fig. 5b and c). Furthermore, evaluation for DNA fragmentation as part of measure to assess oxidative cellular damage revealed that the oral exposure to either AgNPs singly or in combination with Trolox had no detectable adverse effect on rat brain DNA (Fig. 6). Actually, the oral exposure to AgNPs singly and in combination with Trolox showed lower but nonsignificant DNA fragmentation \% compared with the negative control.

\subsection{AgNPs elevated levels of reduced glutathione (GSH) in rat brain and} plasma

In order to preliminarily assess the antioxidant status of experimental animals, level of GSH was determined in rat plasma and tissues. The oral exposure to either AgNPs singly or in combination with Trolox caused elevation $(p<0.05)$ in rat plasma GSH level compared to the negative control (Fig. 7a). In rat liver, GSH level was not appreciably affected by the oral exposure to either AgNPs alone or in combination with Trolox (Fig. 7b). However, rat brain GSH level was significantly elevated following the oral exposure to either AgNPs alone or in combination with Trolox (Fig. 7c).

\subsection{Proposed mechanism for activation of rat brain kynurenine by AgNPs}

Several investigations have established that oxidative stress [13,21] and production of cytokines such as IFN- $\gamma$ [22] have potential to activate the kynurenine pathway, as illustrated in Fig. 8. Meantime, AgNPs is capable of causing oxidative stress and/or producing ROS [10,15], as well as activating the kynurenine pathway [11,23]. However, findings in this work indicate that AgNPs might have activated rat brain kynurenine pathway through alternative route that precludes oxidative stress (Fig. 9).

\section{Discussion}

Although, the use of AgNPs for biomedical purposes is steadily increasing and has been gaining wider attraction [23], the limited knowledge about cellular targets or interaction of nanoparticles
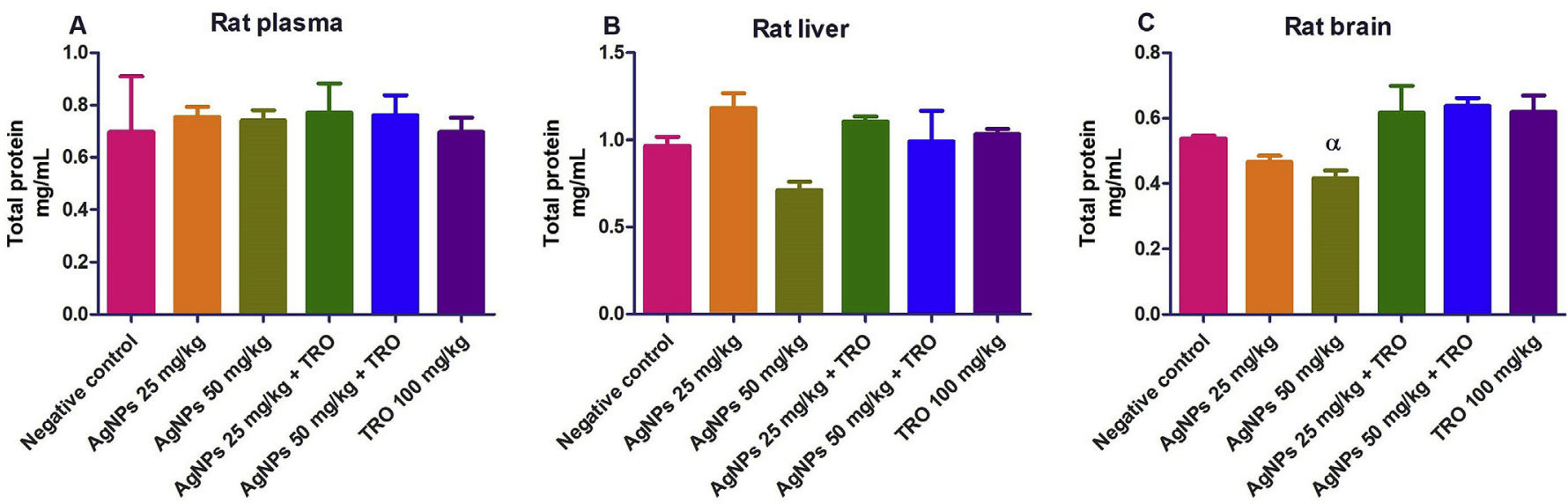

Fig. 2. Effect of oral administration of silver nanoparticles (AgNPs) on rat total protein level; [A] Rat plasma, [B] Rat liver and [C] Rat brain. Data are presented as mean value of four replicates \pm standard error of mean (SEM). $\alpha$ is significant at $p<0.05$ versus AgNPs $(50 \mathrm{mg} / \mathrm{kg}+\mathrm{TRO})$. 

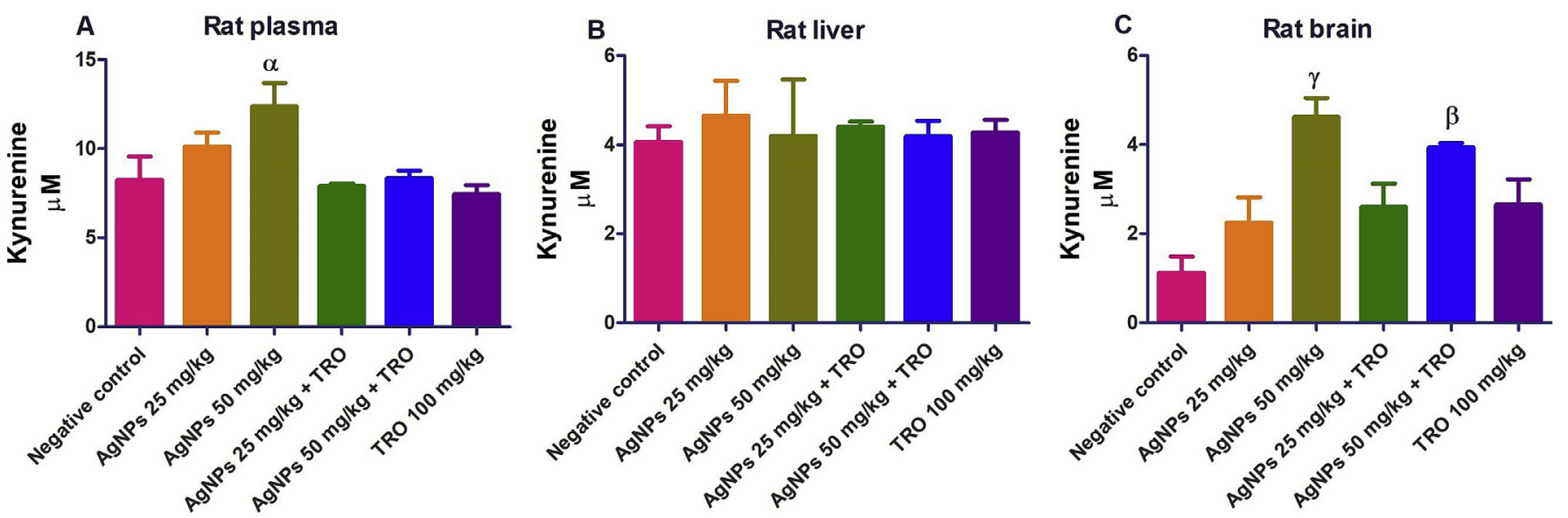

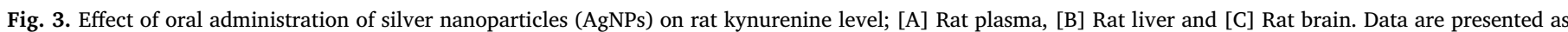

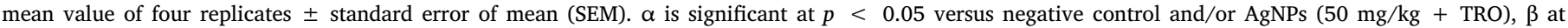
$p<0.001$ versus negative control, $\gamma$ at $p<0.0001$ versus negative control.

remains a challenge that impedes full exploration of the biomedical prospects of nanotechnology. Nonetheless, recent investigations suggest that nanoparticles have multiple cellular targets, among which are the activation of the kynurenine pathway as well as the production of reactive oxygen species (ROS) leading to oxidative stress [1,10,11,23]. Interestingly, ROS and/or oxidative stress has been strongly linked with the oxidative degradation of L-tryptophan to form kynurenine [21]. However, we do not know yet whether AgNPs activates the kynurenine pathway by facilitating oxidative stress and/or ROS production. Therefore, in this work, we examined the involvement of oxidative stress in relation to the activation of the kynurenine pathway by AgNPs in Wistar rats.

Our results showed that AgNPs elevated kynurenine levels in rat plasma and brain and not in the rat liver. This finding is supportive of and confirms our earlier report which demonstrated the capacity of inorganic nanoparticles to activate the kynurenine pathway in vitro [11]. That AgNPs failed to elevate kynurenine levels in rat liver could be that TDO which is majorly responsible for L-tryptophan degradation in liver was not activated and/or inhibited by AgNPs or its metabolites. Meantime, studies have shown that inflammation and oxidative stress are among factors that facilitate the oxidative breakdown of L-tryptophan through the kynurenine pathway [21]. Although, the activation of the kynurenine pathway has been linked with oxidative stress [13], our results in this work indicated that AgNPs might have activated the rat brain kynurenine pathway independently of oxidative stress and/or
ROS production. This is more plausible if we consider that the cotreatment of AgNPs with Trolox failed to appreciably modulate rat brain kynurenine level. That the co-treatment with Trolox antioxidant failed to restore the kynurenine level in rat brain underscores the exclusion of oxidative stress in the activation of the kynurenine pathway by AgNPs. Conversely, the co-treatment with Trolox restored the rat plasma kynurenine level comparable to that of negative control. This finding did not only suggest culpability of oxidative stress in the activation of the rat plasma kynurenine pathway, but also implicated that AgNPs may affect cellular targets differently depending on localization within the cells. This line of thought would conform with and support our earlier report which showed that metal nanoparticles have multiple cellular targets [11]. Considered together, our data indicated that AgNPs might have activated the rat brain kynurenine pathway through an alternative route that precludes oxidative stress. In addition, separate studies have shown that the activation of the kynurenine pathway could be driven by cytokine production, particularly interferon-gamma (IFN- $\gamma$ ) [22]. Although in the present study, we did not investigate IFN$\gamma$ in relation to the activation of the kynurenine pathway by AgNPs, nevertheless, AgNPs is immunogenic [2] and upregulates production of IFN- $\gamma$ [24]. Therefore, it is within the realm of possibility that AgNPs activated rat brain kynurenine viz-a-viz upregulation of IFN- $\gamma$.

Furthermore, our results in this work revealed that the oral exposure to AgNPs might not have caused oxidative stress in rat plasma and tissues; the oxidative stress indices in rat plasma and tissues were not
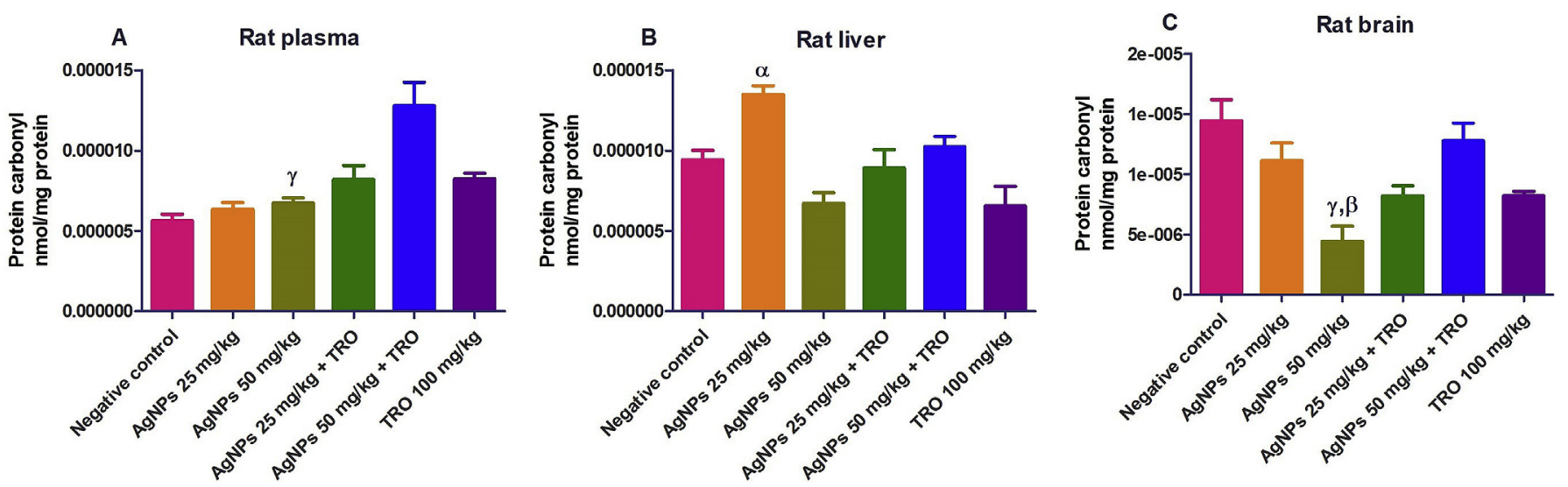

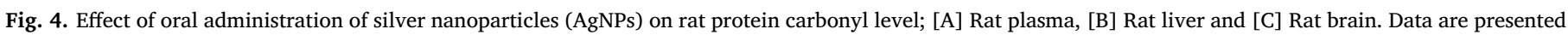

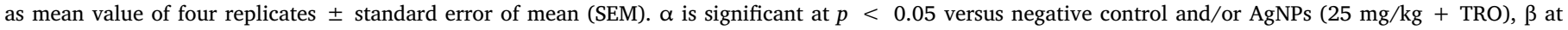
$p<0.001$ versus AgNPs (50 mg/kg + TRO), $\gamma$ at $p<0.0001$ versus negative control and/or AgNPs (50 mg/kg + TRO). 

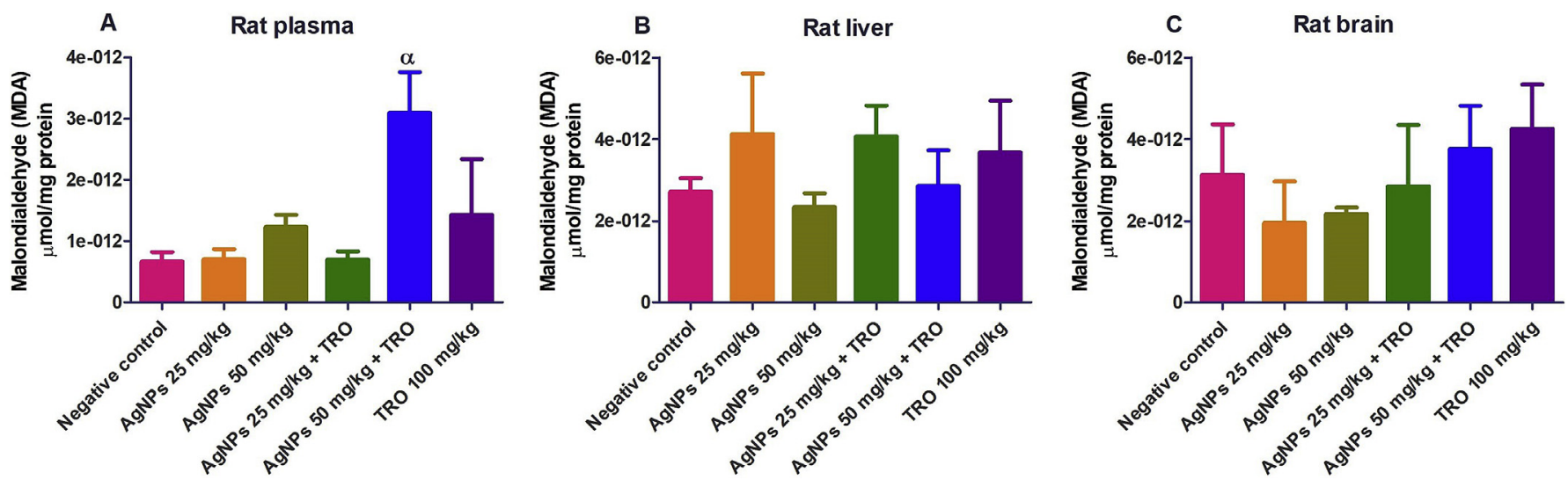

Fig. 5. Effect of oral administration of silver nanoparticles (AgNPs) on rat malondialdehyde (MDA) level; [A] Rat plasma, [B] Rat liver and [C] Rat brain. Data are presented as mean value of four replicates \pm standard error of mean (SEM). $\alpha$ is significant at $p<0.05$ versus negative control.

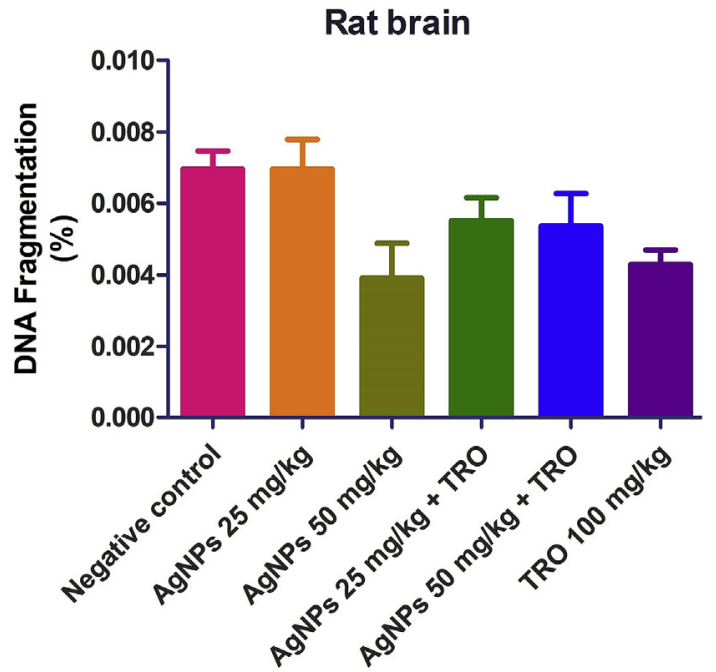

Fig. 6. Effect of oral administration of silver nanoparticles (AgNPs) on rat brain DNA. Data are presented as mean value of four replicates \pm standard error of mean (SEM).

significantly different from those of negative control. Conversely, the co-treatment with AgNPs and Trolox elevated rat plasma and brain levels of lipid peroxidation and protein carbonyl. This finding may indicate a modulating effect of Trolox on AgNPs. It is likely that Trolox

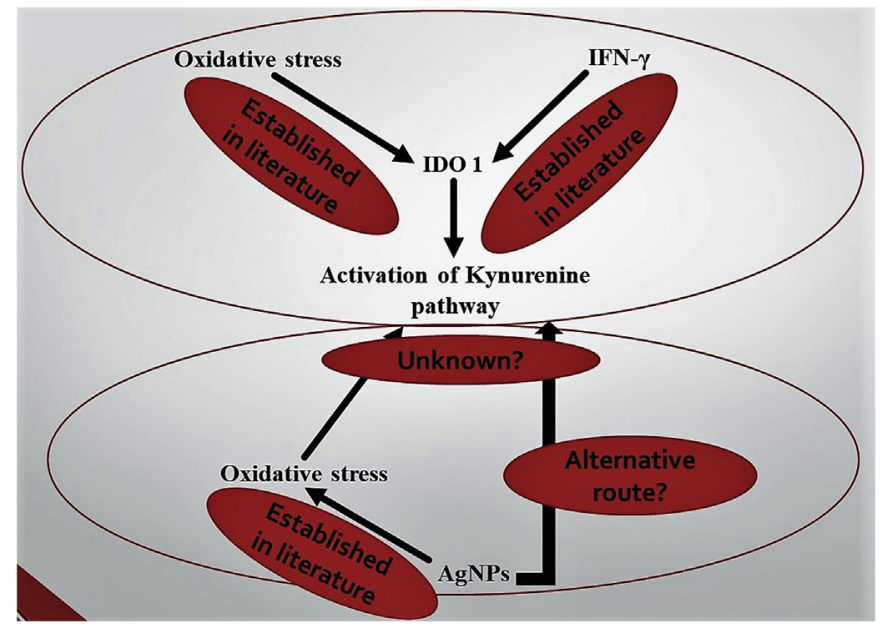

Fig. 8. Illustration of kynurenine activation as demonstrated by existing investigations (Badawy, 2017; Myint and Kim, 2014, Karu et al., 2016).

potentiated the effect of AgNPs towards increasing the indices of oxidative stress in rat plasma and brain. Taken together, data support that AgNPs did not cause oxidative stress at the doses investigated in this study. Moreover, oral exposure to AgNPs alone or in combination with Trolox had no detectable effect on rat brain DNA. Indeed, the oral exposure to AgNPs singly and in combination with Trolox might have
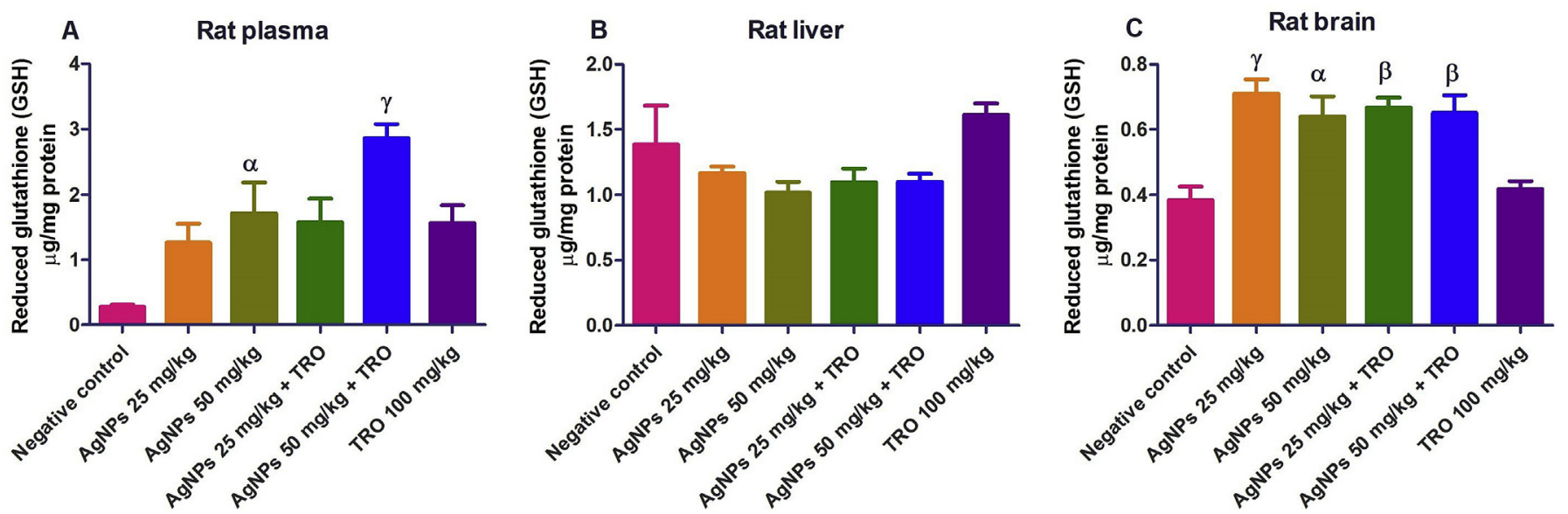

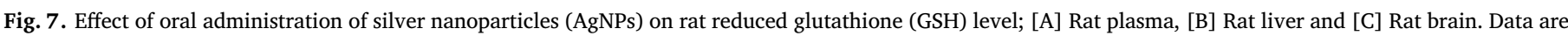

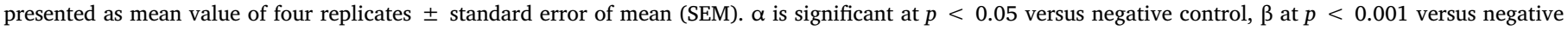
control, $\gamma$ at $p<0.0001$ versus negative control. 


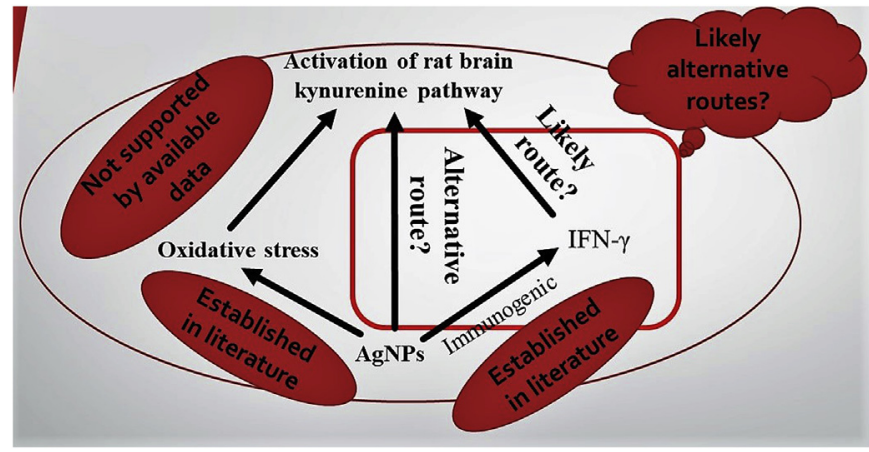

Fig. 9. Proposed mechanistic activation of the rat brain kynurenine pathway by silver nanoparticles (AgNPs) precludes oxidative stress.

protected rat brain DNA against occasional damage as there was lower $\%$ DNA fragmentation recorded compared to the negative control. Additionally, we found that GSH level as marker of antioxidant status was significantly elevated in rat plasma and brain following the oral exposure to AgNPs. The elevation of GSH in rat plasma and brain could be an adaptive mechanism in response to AgNPs exposure. The elevated GSH level in these rat tissues may also explain the absence of oxidative stress. Generally, GSH forms part of the cellular non-enzymatic antioxidant defense system that counteracts effect of free radical assaults $[25,26]$. Therefore, the elevated GSH levels in rat plasma and brain might buffer against likely oxidative stress resulting from the oral exposure to AgNPs.

In conclusion, our data indicate that the oral exposure to AgNPs elevated kynurenine level in rat plasma and brain. Further, the elevation of kynurenine level in rat plasma and not rat brain was susceptible to modulation and amelioration by the co-treatment with Trolox. Taken together, our finding suggests that the activation of the kynurenine pathway in rat brain was independent of oxidative stress. In addition, data also suggest potentiating effect of Trolox on AgNPs exposure in manners that caused oxidative stress in rat plasma and tissues. Taken together, the findings are not only new but will contribute to deepen our understanding of the cellular interaction of nanoparticles.

\section{Conflicts of interest}

The authors report no competing interests.

\section{Acknowledgements}

Authors acknowledge the laboratory staff at the Department of Biochemistry for their technical support.

\section{Transparency document}

Transparency document related to this article can be found online at https://doi.org/10.1016/j.cbi.2019.01.034.

\section{Appendix A. Supplementary data}

Supplementary data to this article can be found online at https:// doi.org/10.1016/j.cbi.2019.01.034.

\section{References}

[1] O.S. Adeyemi, I. Adewumi, T. Faniyan, Silver nanoparticles influenced rat serum metabolites and tissue morphology, J. Basic Clin. Physiol. Pharmacol. 26 (2015) 355-361, https://doi.org/10.1515/jbcpp-2013-0092.

[2] P. Khanna, C. Ong, B. Bay, G. Baeg, Nanotoxicity: an interplay of oxidative stress, inflammation and cell death, Nanomaterials 5 (2015) 1163-1180, https://doi.org/ 10.3390/nano5031163.

[3] O.S. Adeyemi, N.I. Molefe, O.J. Awakan, C.O. Nwonuma, O. Alejolowo, T. Olaolu, R.F. Maimako, K. Suganuma, Y. Han, K. Kato, Metal nanoparticles show potential to restrict Trypanosoma growth, Artif. Cells Nanomed. Biotechnol. (2018 Jul 22) 1-9, https://doi.org/10.1080/21691401.2018.1489267 In press.

[4] C. Yah, G. Simate, Nanoparticles as potential new generation broad spectrum antimicrobial agents, Daru 23 (2015) 43, https://doi.org/10.1186/s40199-0150125-6.

[5] G. Franci, A. Falanga, S. Galdiero, et al., Silver nanoparticles as potential antibacterial agents, Molecules 20 (2015) 8856-8874, https://doi.org/10.3390/ molecules20058856.

[6] O.S. Adeyemi, C.G. Whiteley, Interaction of nanoparticles with arginine kinase from Trypanosoma brucei: kinetic and mechanistic evaluation, Int. J. Biol. Macromol. 62 (2013) 450-456, https://doi.org/10.1016/j.ijbiomac.2013.09.008.

[7] O.S. Adeyemi, C.G. Whiteley, Interaction of metal nanoparticles with recombinant arginine kinase from Trypanosoma brucei: thermodynamic and spectrofluorimetric evaluation, Biochim. Biophys. Acta Gen. Subj. 1840 (2014) 701-706, https://doi. org/10.1016/j.bbagen.2013.10.038.

[8] A. Hudecova, B. Kusznierewicz, E. Runden-Pran, et al., Silver nanoparticles induce premutagenic DNA oxidation that can be prevented by phytochemicals from Gentiana asclepiadea, Mutagenesis 27 (2012) 759-769, https://doi.org/10.1093/ mutage/ges046.

[9] P. Saini, S. Saha, P. Roy, P. Chowdhury, S.P. Sinha Babu, Evidence of reactive oxygen species (ROS) mediated apoptosis in Setaria cervi induced by green silver nanoparticles from Acacia auriculiformis at a very low dose, Exp. Parasitol. 160 (2016) 39-48, https://doi.org/10.1016/j.exppara.2015.11.004.

[10] O.S. Adeyemi, Y. Murata, T. Sugi, K. Kato, Inorganic nanoparticles kill Toxoplasma gondii via changes in redox status and mitochondrial membrane potential, Int. J. Nanomed. 12 (2017) 1647-1661, https://doi.org/10.2147/ijn.s122178.

[11] O.S. Adeyemi, Y. Murata, T. Sugi, Y. Han, K. Kato, Modulation of host HIF-1 $\alpha$ activity and the tryptophan pathway contributes to the anti- Toxoplasma gondii potential of nanoparticles, Biochem. Biophys. Rep. 11 (2017) 84-92, https://doi.org/ 10.1016/j.bbrep.2017.07.004.

[12] J. Sternberg, C. Forrest, R. Dalton, C. Turner, J. Rodgers, T.W. Stone, P.G.E. Kennedy, Kynurenine pathway activation in human African trypanosomiasis, JID (J. Infect. Dis.) 215 (2017) 806-812, https://doi.org/10.1093/infdis/jiw623.

[13] A. Myint, Y. Kim, Network beyond Ido in psychiatric disorders: revisiting neurodegeneration hypothesis, Prog. Neuro Psychopharmacol. Biol. Psychiatr. 48 (2014) 304-313, https://doi.org/10.1016/j.pnpbp.2013.08.008.

[14] Council for International Organizations of Medical Sciences, International guiding Principles for biomedical Research involving animals issued by CIOMS, Vet. Q. 8 (1985) 350-352, https://doi.org/10.1080/01652176.1986.9694068.

[15] O.S. Adeyemi, T. Faniyan, Antioxidant status of rats administered silver nanoparticles orally, J. Taibah Univ. Med. Sci. 9 (2014) 182-186, https://doi.org/10. 1016/j.jtumed.2014.03.002.

[16] A. Gornall, C. Bardawill, M. David, Determination of serum proteins by means of the biuret reaction, J. Biol. Chem. 177 (1949) 751-766.

[17] A. Castegna, J. Drake, C. Pocernich, D. Butterfield, Protein carbonyl levels-an assessment of protein oxidation, in: K. Hensley, R. Floyd (Eds.), Methods in Pharmacology and Toxicology: Methods in Biological Oxidative Stress, Humana Press, Totowa NJ, 2003, pp. 161-168.

[18] R. Varshney, R. Kale, Effects of calmodulin antagonists on radiation-induced lipid peroxidation in microsomes, Int. J. Radiat. Biol. 58 (1990) 733-743, https://doi. org/10.1080/09553009014552121.

[19] E. Bentler, O. Duran, K. Mikus, Improved method for determination of blood glutathione, J. Lab. Clin. Med. 61 (1963) 882-888.

[20] C. Perandones, V. Illera, D. Peckham, L.L. Stunz, R.F. Ashman, Regulation of apoptosis in vitro in mature murine spleen T cells, J. Immunol. 151 (1993) 3521-3529.

[21] N. Karu, C. McKercher, D. Nichols, N. Davies, R.A. Shellie, E.F. Hilder, M.D. Jose, Tryptophan metabolism, its relation to inflammation and stress markers and association with psychological and cognitive functioning: tasmanian Chronic Kidney Disease pilot study, BMC Nephrol. 17 (2017) 171, https://doi.org/10.1186/s12882016-0387-3.

[22] A.A. Badawy, Kynurenine pathway of tryptophan metabolism: regulatory and functional aspects, Int. J. Tryptophan Res. 10 (2017), https://doi.org/10.1177/ 1178646917691938 10:117864691769193.

[23] O.S. Adeyemi, Y. Murata, T. Sugi, Y. Han, K. Kato, Exploring amino acid-capped nanoparticles for selective anti-parasitic action and improved host biocompatibility, J. Biomed. Nanotechnol. 14 (2018) 847-867, https://doi.org/10.1166/jbn.2018. 2544.

[24] Y. Luo, L. Chang, P. Lin, Metal-based nanoparticles and the immune system: activation, inflammation, and potential applications, BioMed Res. Int. (2015) 1-12, https://doi.org/10.1155/2015/143720.

[25] O.S. Adeyemi, F.A. Sulaiman, Biochemical and morphological changes in Trypanosoma brucei brucei- infected rats treated with homidium chloride and diminazene aceturate, J. Basic Clin. Physiol. Pharmacol. 23 (2012) 179-183, https:// doi.org/10.1515/jbcpp-2012-0018.

[26] O.S. Adeyemi, O.I. Olajide, A.A. Adeyanju, O.J. Awakan, D.A. Otohinoyi, Modulation of rat plasma kynurenine level by platinum nanoparticles and likely association with oxidative stress, Biointerface Res. Appl. Chem. 8 (2018) 3364-3367. 